\title{
Vitamin E reduces hepatic fibrosis in mice with Schistosoma japonicum infection
}

\author{
XUEFENG WANG, RONGBO ZHANG, JIUWEI DU, YOUYING HU, LIFA XU, JUN LU and SONG YE
}

\author{
Department of Pathogen Biology and Immunology, School of Medicine, \\ Anhui University of Science and Technology, Anhui 232001, P.R. China
}

Received August 11, 2011; Accepted October 25, 2011

DOI: $10.3892 / \mathrm{mmr} .2011 .654$

\begin{abstract}
To investigate whether vitamin E protects against hepatic fibrosis in mice with Schistosoma japonicum infection, 24 pathogen-free Kunming mice were selected and randomly divided into four groups: control (uninfected, untreated), model (infected, untreated), low-dose intervention (infected, vitamin E-treated, $30 \mathrm{mg} / \mathrm{g}$ bodyweight/day) and high-dose intervention (infected, vitamin E-treated, $60 \mathrm{mg} / \mathrm{g}$ bodyweight/day). Mice were infected with Schistosoma japonicum by inoculating abdominal skin with snail hosts. The activities of malondialdehyde (MDA), superoxide dismutase (SOD), glutathione peroxidase (GPx) and catalase (CAT) were detected in hepatic tissue by colorimetry. The expression levels of laminin (LN), hyaluronic acid (HA), procollagen type III (PC-III) and type IV collagen (IV-C) were detected in the serum by radioimmunoassay. Finally, areas and numbers of granulomas were assessed through histopathology 42 days following treatment. The results revealed that mean areas of granulomas were smaller in the low- and high-dose intervention groups compared to those in the model group. Furthermore, the higher dose of vitamin $\mathrm{E}$ resulted in smaller granulomas than the low dose. The levels of LN, HA, PC-III and IV-C in the serum were lower following vitamin $\mathrm{E}$ treatment than in the model group. By contrast, activity of SOD, GPx and CAT in hepatic tissue was higher following vitamin E treatment compared to the model group. The activity of MDA was lower in hepatic tissue following vitamin E treatment compared to the model group, but was higher compared to controls. In general, the higher dose of vitamin $\mathrm{E}$ affected measurements to a greater
\end{abstract}

Correspondence to: Dr Xuefeng Wang, Department of Pathogen Biology and Immunology, School of Medicine, Anhui University of Science and Technology, Dongshanzhong Road 25, Huainan, Anhui 232001, P.R. China

E-mail: wangxxff1023@126.com

Abbreviations: MDA, malondialdehyde; SOD, superoxide dismutase; GPx, glutathione peroxidase; CAT, catalase; LN, laminin; HA, hyaluronic acid; PC-III, procollagen type III; IV-C, type IV collagen

Key words: Schistosoma japonicum, hepatic fibrosis, superoxide dismutase, glutathione peroxidase, malondialdehyde, granulation nodus extent than the lower dose. In conclusion, vitamin E treatment may reduce the growth of granulomas, slowing the process of hepatic fibrosis, and this effect may be the result of the altered activity of the oxidation-reduction enzyme system.

\section{Introduction}

Schistosomiasis, a common parasitic disease in tropical and subtropical regions, currently affects more than 600 million individuals worldwide (1). This infectious disease occurs in southern regions of China and, despite extensive preventative measures, there remain epidemic areas in the country (2). One significant effect of schistosome infection is liver damage, mainly resulting from egg-induced granuloma formation and progressive hardening of the liver (fibrosis) (3). As with other forms of liver disease, this damage occurs through immune responses, oxidative stress and other mechanisms (4). Studies of other liver diseases have shown that antioxidants, which alter oxidoreductase activities, may improve symptoms (5). One antioxidant, vitamin E, has shown some efficacy in treating liver damage caused by infection with other Schistosoma species (6). Here, we investigated the effects of vitamin $\mathrm{E}$ on liver fibrosis in schistosome-infected mice.

\section{Materials and methods}

Experimental animals. A total of 36 female Kunming mice (pathogen-free), 6-weeks-old and weighing $25( \pm 2) \mathrm{g}$, were purchased from the Nanjing Medical University Animal House (China). Mice were randomized to four groups, with 8 mice per group: control, model, low-dose intervention and high-dose intervention. The model, low-dose intervention and high-dose intervention groups were infected with schistosome cercariae through the abdominal skin $(\sim 25 \pm 2$ cercariae per mouse). Control mice were not infected. Mice in the low-dose and highdose intervention groups were administered vitamin $\mathrm{E}$ at 30 and $60 \mathrm{mg} / \mathrm{day} / \mathrm{kg}$, respectively, from the day of infection. Vitamin E was dissolved in $2 \mathrm{ml}$ normal saline and administered intragastrically. Mice in the control and model groups were administered an equivalent volume of normal saline by intragastric administration. Treatment occurred every day for 42 days; subsequently, all mice were sacrificed for analysis. Blood samples were collected by retro-orbital bleeding. Following opening up of the abdomen, the whole livers were removed. Hepatic middle lobule 
slices were harvested and remaining liver tissue was cryopreserved for subsequent studies. Snails infected with schistosome cercariae were obtained from the Hunan Institute of Parasitic Disease, and animal surgical instruments and equipment were from Nanjing Medical University Animal House.

Liver tissue homogenates. Liver tissue was thawed and rinsed, then cut into pieces. Tissue $(0.2 \mathrm{~g})$ was placed into a $10-\mathrm{ml}$ beaker. A volume of homogenate solution $(0.1 \mathrm{mM}$ Tris-HCL, $0.01 \mathrm{mM}$ EDTA-2Na, $0.01 \mathrm{mM}$ sucrose, $0.8 \% \mathrm{NaCl}$ ) nine times $(\mathrm{w}: \mathrm{v}=1: 4)$ the amount of tissue was added. Liver was processed with a homogenizer for 6-8 $\mathrm{min}$ in order to fully homogenize the pieces. Samples were centrifuged at $3,000 \mathrm{x}$ g at $4^{\circ} \mathrm{C}$ for 10-15 min. Appropriate amounts of clear supernatant liquid were used for protein detection.

Malondialdehyde (MDA), superoxide dismutase (SOD), glutathione peroxidase (GPx) and catalase (CAT) detection. MDA, SOD, GPx and CAT activity in liver tissue homogenates was detected by colorimetry according to the manufacturer's instructions. Concentrations were calculated by standard curve. MDA, SOD, GPx and CAT assay kits were obtained from the Nanjing Jiancheng Bioengineering Institute (Nanjing, China).

Laminin (LN), hyaluronic acid (HA), procollagen type III (PC-III) and type IV collagen $(I V-C)$ detection. The LN, HA, $\mathrm{PC}-\mathrm{III}$ and IV-C concentrations in liver tissue homogenates were detected by radioimmunoassay according to the manufacturer's instructions. LN, HA, PC-III and IV-C assay kits were provided by the Tianjin Atomic Energy Industry (Tianjin, China)

Liver pathology. The middle lobule of the liver was fixed in $4 \%$ formaldehyde, embedded in paraffin, sectioned at a $4-\mu \mathrm{m}$ thickness and collected onto glass slides. Paraffin-embedded samples were dewaxed and stained with H\&E. In order to determine the number and size of granulomas, five different visual fields were captured at x400 magnification. An image analysis system (Ventana Medical Systems, Inc., Tucson, AZ, USA) was applied to measure the granuloma area.

Statistical methods. SPSS13.0 statistical software was used to analyze data. Analysis of variance was used to compare results, with inter-group comparison performed by the SLN-q test. The $\alpha$ level was set at 0.05 . All values are expressed as the means \pm standard deviation.

\section{Results}

Vitamin E treatment improves liver pathology in infected mice. Livers from mice in the control group were full, brown, with sharp edges, soft texture, clear lobules and normal structure under microscopic examination. The portal and hepatic sinus appeared normal with uniform distribution (Fig. 1A). Mice in the model group had enlarged livers with pale nodular bulges on the surface. Microscopic examination revealed granulomas containing eggs, inflammatory infiltration and surrounding fibroplastic proliferation. Partial nodule edges were irregular, and partial hepatic sinus and portal areas were pressed, twisted and deformed, with more inflammatory cells in the perisinusoidal space (Fig. 1B). Compared to the model group, livers
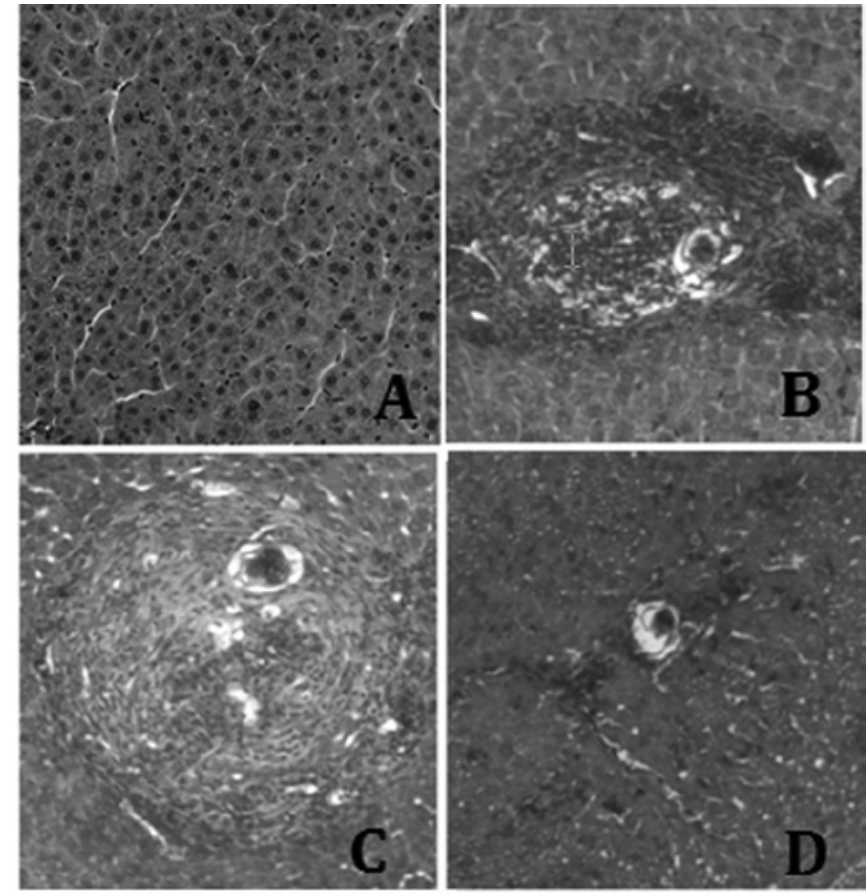

Figure 1. H\&E-stained liver tissue from mice infected with Schistosoma. (A) Control group; (B) model group; (C) low-dose group; (D) high-dose group. Granuloma formation is present in the model, low-dose and high-dose groups.

Table I. Granuloma number and area in animal models of schistosomiasis treated with vitamin E.

\begin{tabular}{lccc}
\hline $\begin{array}{l}\text { Treatment } \\
\text { group }\end{array}$ & $\mathrm{n}$ & $\begin{array}{c}\text { Mean no. of } \\
\text { granulomas }\end{array}$ & $\begin{array}{c}\text { Mean area of } \\
\text { granuloma }\left(\mathrm{mm}^{2}\right)\end{array}$ \\
\hline Control & 8 & $0.0 \pm 0.00$ & $0.0 \pm 0.00$ \\
Model & 8 & $12.9 \pm 1.36^{\mathrm{a}}$ & $6.1 \pm 0.89^{\mathrm{a}}$ \\
Low-dose & 8 & $11.8 \pm 2.18^{\mathrm{a}}$ & $4.5 \pm 0.75^{\mathrm{a}, \mathrm{b}}$ \\
High-dose & 8 & $11.4 \pm 2.20^{\mathrm{a}}$ & $2.7 \pm 0.78^{\mathrm{a}, \mathrm{b}, \mathrm{c}}$ \\
$\mathrm{F}$ & & 101.62 & 111.13 \\
$\mathrm{P}$ & & 0.00 & 0.00 \\
\hline
\end{tabular}

${ }^{\mathrm{a}} \mathrm{P}<0.05$ vs. the control group; ${ }^{\mathrm{b}} \mathrm{P}<0.05$ vs. the model group; ${ }^{\mathrm{P}}<0.05$ vs. the low-dose group.

from mice receiving low- and high-dose vitamin $\mathrm{E}$ intervention had fewer pale nodular bulges on the liver surface and less deformation. Furthermore, microscopic examination revealed egg-containing granulomas, inflammatory infiltration and surrounding fibroplastic proliferation. In the two treatment groups, partial nodule edges were irregular, and partial hepatic sinus and portal areas were pressed, twisted and deformed, with more inflammatory cells in the perisinusoidal space and hepatic sinus (Fig. 1C and D).

Granuloma number and areas were improved in infected mice receiving vitamin $E$ treatment. Livers from control (uninfected and untreated) mice exhibited no granulomas; therefore, the mean numbers and areas of granulomas in each of the infected groups were significantly increased over the controls (Table I). However, the mean areas of granulomas in the low- and 
Table II. Concentrations of LN, HA, PC-III and IV-C in liver tissue homogenates from vitamin E-treated and untreated mice.

\begin{tabular}{|c|c|c|c|c|c|}
\hline Groups & $\mathrm{n}$ & LN (ng/ml) & HA (ng/ml) & PC-III (ng/ml) & IV-C (ng/ml) \\
\hline Control & 8 & $53.9 \pm 6.36$ & $708.9 \pm 29.54$ & $72.0 \pm 6.59$ & $32.0 \pm 5.95$ \\
\hline Model & 8 & $89.5 \pm 8.32^{\mathrm{a}}$ & $1,466.9 \pm 124.40^{\mathrm{a}}$ & $122.4 \pm 9.72^{\mathrm{a}}$ & $45.5 \pm 3.78^{a}$ \\
\hline Low-dose & 8 & $75.6 \pm 6.25^{\mathrm{a}, \mathrm{b}}$ & $1,254.8 \pm 121.62^{\mathrm{a}, \mathrm{b}}$ & $91.1 \pm 8.45^{\mathrm{a}, \mathrm{b}}$ & $41.8 \pm 3.65^{\mathrm{a}}$ \\
\hline High-dose & 8 & $70.8 \pm 11.59^{\mathrm{a}, \mathrm{b}}$ & $1,003.6 \pm 143.63^{\mathrm{a}, \mathrm{b}, \mathrm{c}}$ & $83.9 \pm 7.14^{\mathrm{a}, \mathrm{b}}$ & $36.0 \pm 4.07^{\mathrm{a}, \mathrm{b}}$ \\
\hline $\mathrm{F}$ & & 24.46 & 66.04 & 56.90 & 17.05 \\
\hline $\mathrm{P}$ & & 0.00 & 0.00 & 0.00 & 0.00 \\
\hline
\end{tabular}

${ }^{\mathrm{a}} \mathrm{P}<0.05$ vs. the control group; ${ }^{\mathrm{b}} \mathrm{P}<0.05$ vs. the model group; ${ }^{\mathrm{c}} \mathrm{P}<0.05$ vs. the low-dose group. $\mathrm{LN}$, laminin; HA, hyaluronic acid; $\mathrm{PC}-\mathrm{III}$, procollagen type III; IV-C, type IV collagen.

Table III. Concentrations of MDA, SOD, GPx and CAT in liver tissue homogenates from vitamin E-treated or untreated models of schistosomiasis.

\begin{tabular}{|c|c|c|c|c|c|}
\hline Groups & $\mathrm{n}$ & MDA (U/mg) & $\mathrm{SOD}(\mathrm{U} / \mathrm{mg})$ & GPx (U/mg) & CAT(U/mg) \\
\hline Control & 8 & $2.6 \pm 0.48$ & $171.9 \pm 9.43$ & $41.2 \pm 2.55$ & $18.7 \pm 5.33$ \\
\hline Model & 8 & $5.8 \pm 0.54^{\mathrm{a}}$ & $66.6 \pm 9.77^{a}$ & $16.5 \pm 2.46^{\mathrm{a}}$ & $6.65 \pm 1.12^{\mathrm{a}}$ \\
\hline Low-dose & 8 & $4.6 \pm 0.56^{\mathrm{a}, \mathrm{b}}$ & $93.1 \pm 11.43^{\mathrm{a}, \mathrm{b}}$ & $23.2 \pm 2.72^{\mathrm{a}, \mathrm{b}}$ & $10.85 \pm 1.79^{\mathrm{a}, \mathrm{b}}$ \\
\hline High-dose & 8 & $3.8 \pm 0.37^{\mathrm{a}, \mathrm{b}, \mathrm{c}}$ & $134.6 \pm 13.7^{\mathrm{a}, \mathrm{b}, \mathrm{c}}$ & $30.2 \pm 3.31^{\mathrm{a}, \mathrm{b}, \mathrm{c}}$ & $13.6 \pm 1.51^{\mathrm{a}, \mathrm{b}}$ \\
\hline $\mathrm{F}$ & & 60.33 & 136.31 & 114.80 & 136.31 \\
\hline $\mathrm{P}$ & & 0.00 & 0.00 & 0.00 & 0.00 \\
\hline
\end{tabular}

${ }^{\mathrm{a}} \mathrm{P}<0.05$ vs. the control group; ${ }^{\mathrm{b}} \mathrm{P}<0.05$ vs. the model group; ${ }^{\mathrm{P}}<0.05$ vs. the low-dose group. MDA, malondialdehyde; $\mathrm{SOD}$, superoxide dismutase; GPx, glutathione peroxidase; CAT, catalase.

high-dose intervention groups were smaller than those in the model group. Furthermore, the higher dose of vitamin E resulted in even smaller granulomas than the lower dose.

Vitamin E lowers markers of liver fibrosis in infected mice. To better measure liver fibrosis, we measured the concentrations of several markers (Table II). LN, HA, PC-III and IV-C concentrations were elevated in each of the infected groups compared to controls. However, in mice receiving vitamin $\mathrm{E}$ treatment, each of these markers exhibited reduced levels compared to the model group. Furthermore, the high-dose intervention group had lower concentrations of HA and IV-C than the low-dose intervention group.

Oxidoreductase enzymes are altered following vitamin E treatment. To gain an understanding of the potential mechanism behind the observed improvements in liver fibrosis, we measured markers of oxidoreductase activity in liver tissue (Table III). The concentrations of MDA detected in liver samples from all infected groups were significantly higher compared to the control liver samples. However, MDA levels were highest in the model group and significantly lower than the model group in the two vitamin E treatment groups. By contrast, concentrations of SOD, GPx and CAT detected in liver samples from all infected groups were significantly lower than in control liver samples. However, levels of SOD, GPx and CAT were lowest in the model group, and vitamin $\mathrm{E}$ treatment resulted in significant increases in these levels compared to the model group.

\section{Discussion}

Schistosomiasis is a parasitic disease that infects a multitude of host species, including humans. The disease originates from several Schistosoma species, including Schistosoma mansoni, Schistosoma haematobium and Schistosoma japonicum. The most common of these in China is Schistosoma japonicum, which, despite recent preventative measures, still infects more than 800,000 individuals a year (7).

Schistosomiasis causes progressive liver damage. Schistosoma eggs implant in the liver sinus and, with marked antigenic characteristics, cause local and systemic immune responses $(8,9)$. The eggs stimulate granuloma formation and lead to progressive liver fibrosis. Indeed, over time, the eggs, which continuously release antigens, activate hepatic stellate cells (HSCs), only increasing granuloma formation and exacerbating fibrosis. Furthermore, granulomatous nodules may impede the liver portal system, causing severe portal hypertension with bleeding and liver failure.

Schistosome-induced liver fibrosis is closely linked to the immune response. Egg antigens induce imbalance in T-cell immunity, thought to be a trigger of liver fibrosis (10). In addition, schistosome infection causes imbalance of Th1/Th2 cell immunity, excessive activation of Th2 and activation of HSCs into muscle-like fibroblasts. These fibroblasts increase collagen formation and suppress its decomposition, which eventually produces matrix protein deposition and fibrosis. Th2-derived IL-13 and IL-4 cytokines cause liver damage and 
proliferation of fibrous tissue $(11,12)$, accelerating fibrosis through activation of macrophages and induction of TNF- $\alpha$ and other inflammatory cytokines.

Oxidative stress also plays a significant role in the process of liver fibrosis. A previous study (13) reported that lack of oxygen stimulates HSC activity and promotes expression of vascular endothelial growth factor and proliferation of collagen. In schistosome-induced liver damage, abnormal expression of MDA, SOD and other redox factors was found to promote liver fibrosis. Increased levels of MDA indicate lipid peroxidation, which may activate HSCs and directly stimulate collagen gene transcription (14). SOD protects cell structure and function and prevents liver fibrosis by eliminating superoxide anions produced during lipid peroxidation (15). In animal models of schistosomiasis, oxygen-free radical damage leads to liver fibrosis $(16,17)$. Thus, it is hypothesized that interventions affecting the activity of the oxidative stress system may effectively alleviate liver injury.

One source of intervention is fat-soluble vitamins with strong reducing activity, which may block free-radical oxidation and lipid peroxidation. Vitamin E, one of the most abundant fat-soluble antioxidants in the human body (18), is capable of protecting membrane lipids from damage caused by oxygen free radicals (19). In a study of carbon tetrachlorideinduced liver fibrosis, vitamin E blocked oxidative stress and alleviated liver damage (20). Here, we investigated the effects of vitamin $\mathrm{E}$ on liver pathology in schistosome-infected mice. Vitamin E treatment reduced the effects of schistosome infection on the liver. While granuloma formation occurred with the same frequency as in untreated infected animals, the size of granulomas was significantly reduced in those treated with vitamin E. In addition, serum indicators of liver fibrosis (LN, HA, PC-III and IV-C) were significantly lower in infected animals treated with vitamin $\mathrm{E}$ than in untreated infected animals and, indeed, this effect was dose-dependent.

We also investigated the potential mechanism for this reduced progression of liver fibrosis by assessing markers of oxidative stress in livers from untreated and treated mice. MDA, which is typically increased in liver fibrosis, was reduced in vitamin E-treated mice compared to untreated infected mice, suggesting a reduced level of lipid peroxidation. Similarly, levels of the antioxidant enzymes SOD, GPx and CAT increased in the infected animals treated with vitamin $\mathrm{E}$ compared to the untreated infected animals, indicating that vitamin E treatment stimulated antioxidant activity. This antioxidant activity may be responsible for the less severe liver pathology observed in infected animals treated with vitamin $\mathrm{E}$ compared to untreated infected animals.

In conclusion, vitamin $\mathrm{E}$ treatment appears to stimulate antioxidant activity within the liver in order to fight fibrosis following schistosome infection. Most measurements were markedly improved in the high-dose intervention group, suggesting that a high dose of vitamin E may slow progression of liver fibrosis in individuals affected by schistosomiasis.

\section{Acknowledgements}

This work was supported by a grant from the National Natural Science Foundation of China (NSFC 30801046), and the grant 2010YB004 from the Anhui University of Science and Technology.

\section{References}

1. Wilson MS, Mentink-Kane MM, Pesce JT, Ramalingam TR, Thompson R and Wynn TA: Immunopathology of schistosomiasis. Immunol Cell Biol 85: 148-154, 2007.

2. Zhu R, Gray DJ, Thrift AP, et al: A 5-year longitudinal study of schistosomiasis transmission in Shian Village, the Anning River Valley, Sichuan Province, the People's Republic China. Parasit Vectors 4: 43-45, 2011.

3. Pearce EJ and MacDonald AS: The immunobiology of schistosomiasis. Nat Rev Immunol 2: 499-511, 2002.

4. Elsammak MY, Al-Sharkaweey RM, Ragab MS, Amin GA and Kandil MH: IL-4 and reactive oxygen species are elevated in Egyptian patients affected with schistosomal liver disease. Parasite Immunol 30: 603-609, 2008.

5. Ha HL, Shin HJ, Feitelson MA and Yu DY: Oxidative stress and antioxidants in hepatic pathogenesis. World J Gastroenterol 16: 6035-6043, 2010.

6. Khaled IA,EI-Ansary MS, Saleh AF, Mahmoud OM, Baioumi EA and Bakr HA: Cytogenetic study of the effect of Schistosoma mansoni infection on human peripheral blood lymphocytes and the role of $\beta$-carotene and vitamin $\mathrm{E}$ in modulating this effect. Mol Biol Rep 38: 4101-4109, 2011.

7. Zhou XN, Wang LY, Chen MG, Wu XH, Jiang QW, Chen XY, Zheng J and Utzinger J: The public health significance and control of schistosomiasis in China - then and now. Acta Tropica 96: 97-105, 2005

8. Chavarria A, Fleury A, Bobes RJ, Morales J, Fragoso G and Sciutto E: A depressed peripheral cellular immune response is related to symptomatic neurocysticercosis. Microbes Infect 8: 1082-1089, 2006.

9. Alvarez JI, Rivera J and Teale JM: Differential release and phagocytosis of tegument glycoconjugates in neurocysticercosis: implications for immune evasion strategies. PLOS Neg Trop Dis 2: e218, 2008.

10. Coutinho HM, Acosta LP, Wu HW, McGarvey ST, Langdon GC, Jiz MA, Jarilla B, Olveda RM, Friedman JF and Kurtis JD: Th2 cytokines are associated with persistent hepatic fibrosis in human Schistosoma japonicum infection. J Infect Dis 195: 288-295, 2007.

11. MacDonald TT: Decoy receptor springs to life and eases fibrosis. Nat Med 12: 13-14, 2006.

12. Fichtner-Feigl S, Strober W, Kawakami K, Puri PK and Kitani A: IL-13 signaling through the IL-13 a2 receptor is involved in induction of TGF- $\beta$ production and fibrosis. Nat Med 12: 99-106, 2006.

13. Vanheule E, Geerts AM, van Huysse J, Schelfhout D, Praet M, van Vlierberghe H, De Vos M and Colle I: An intravital microscopic study of the hepatic microcirculation in cirrhotic mice models: relationship between fibrosis and angiogenesis. Int J Exp Pathol 89: 419-432, 2008.

14. Shi HY, Xu JW and Ren XX: Effect of genistein on hepatic stellate cell proliferation and lipid peroxidation in vitro. Nan Fang Yi Ke Da Xue Xue Bao 28: 2066-2068, 2008.

15. Karadeniz G, Acikgoz S, Tekin IO, Tascylar O, Gun BD and Cömert M: Oxidized low-density-lipoprotein accumulation is associated with liver fibrosis in experimental cholestasis. Clinics 63: 531-540, 2008 .

16. Gharib B, Abdallahi OM, Dessein H and De Reggi M: Development of eosinophil peroxidase activity and concomitant alteration of the antioxidant defense in the liver of mice infected with Schistosoma mansoni. J Hepatol 30: 594-602, 1999.

17. Abdallahi OM, Hanna S, De Reggi M and Gharib B: Visualization of oxygen radical production in mouse liver in response to infection with Schistosoma mansoni. Liver 19: 495-500, 1999.

18. Asemi Z, Taghizadeh M, Sarahroodi S, Jazayeri S, Tabasi Z and Seyyedi F: Assessment of the relationship of vitamin D with serum antioxidant vitamins $\mathrm{E}$ and $\mathrm{A}$ and their deficiencies in Iranian pregnant women. Saudi Med J 31: 1119-1123, 2010.

19. Hong JH, Kim MJ, Park MR, Kwang OG, Lee IS, Byun BH, Lee SC, Lee KB and Rhee SJ: Effects of vitamin E on oxidative stress and membrane fluidity in brain of streptozotocin-induced diabetic rats. Clin Chim Acta 340: 107-115, 2004.

20. An J, Feng GG, Huang L, Kurokawa T, Nonami T, Koide T, Kondo F, Komatsu T, Tsunekawa K, Fujiwara Y, Goto H, Nishikawa H, Miki T, Sugiyama S and Ishikawa N: Effects of 1-O-hexyl-2,3,5-trimethylhydroquinone on carbon tetrachloride-induced hepatic cirrhosis in rats. Hepatol Res 40: 566-573, 2010. 